\title{
The development of a deflectometer for accurate surface figure metrology
}

Mikhail Gubarev, Andrew EberhardtcBrian Ramsey, Carolyn Atkins,

${ }^{a}$ NASA Marshall Space Flight Center, Huntsville, AL (United States)

University of Alabama in Huntsvile, Huntsville, AL (United States) "University of Washington, Seattle, WA (United States)

Introduction

Marshall Space Flight Center is developing the method of direct fabrication for high resolution full-shell x-ray optics. In this technique the $\mathrm{x}$-ray optics axial profiles are figured and polished using a computer-controlled Zeeko IRP600X polishing machine (shown in figure 1). Based on the Chandra optics fabrication history about one third of the manufacturing time is spent on moving a mirror between fabrication and metrology sites, reinstallation and alignment with either the metrology or fabrication instruments. Also, the accuracy of the alignment significantly affects the ultimate accuracy of the resulting mirrors. In order to achieve higher convergence rate it is highly desirable to have a metrology technique capable of in situ surface figure measurements of the optic under fabrication, so the overall fabrication costs would be greatly reduced while removing the surface errors due to the realignment necessary after each metrology cycle during the fabrication. The goal of this feasibility study is to demonstrate if the Phase Measuring Deflectometry can be applied for in situ metrology of full shell x-ray optics. Examples of the full-shell mirror substrates suitable for the direct fabrication are shown in figure 2.
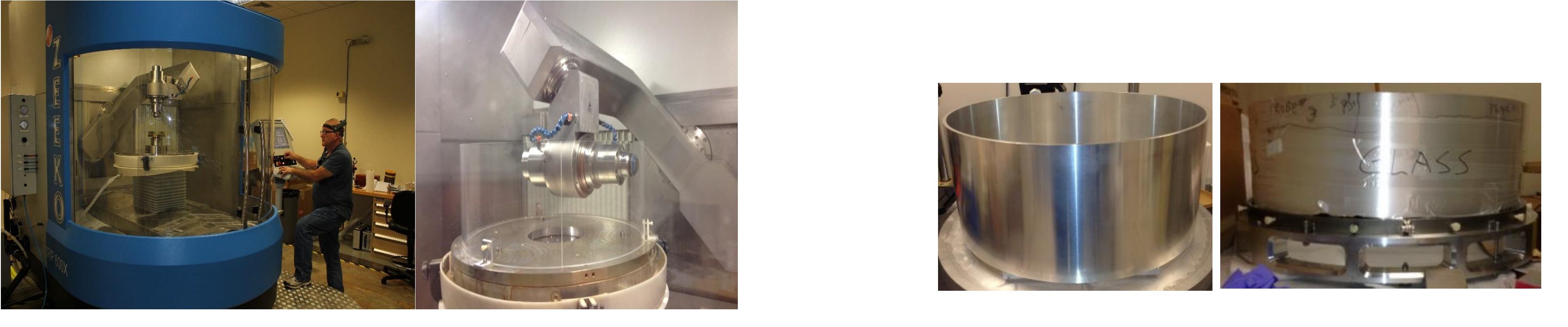

Figure 1. Zeeko IRP600X computer-controlled polishing "bonnet" tool installed inside a plastic shell (right).

Figure 2. Aluminum (left) and glass (right)
substrates for the direct fabrication of high substrates for the direct

Phase Measuring Deflectometry

The concept here is as follows: a perfect fringe pattern disphyed by a monitor is observed after refection from the surface under the test by a camera as shown in figure 1. Deviations from perfect spacing of the observed fringe pattern measured at multiple phases would provide unambiguous measurement of deviations in the slopes of the mirror surface from its ideal shape. Algorithm steps:

Generation of "perfect sinusoidal fringe pattern

Phase recovery using phase shifting

Generating the reference phase map

Unwrapping the phase difference

Slope profile recovery by slope profile integration

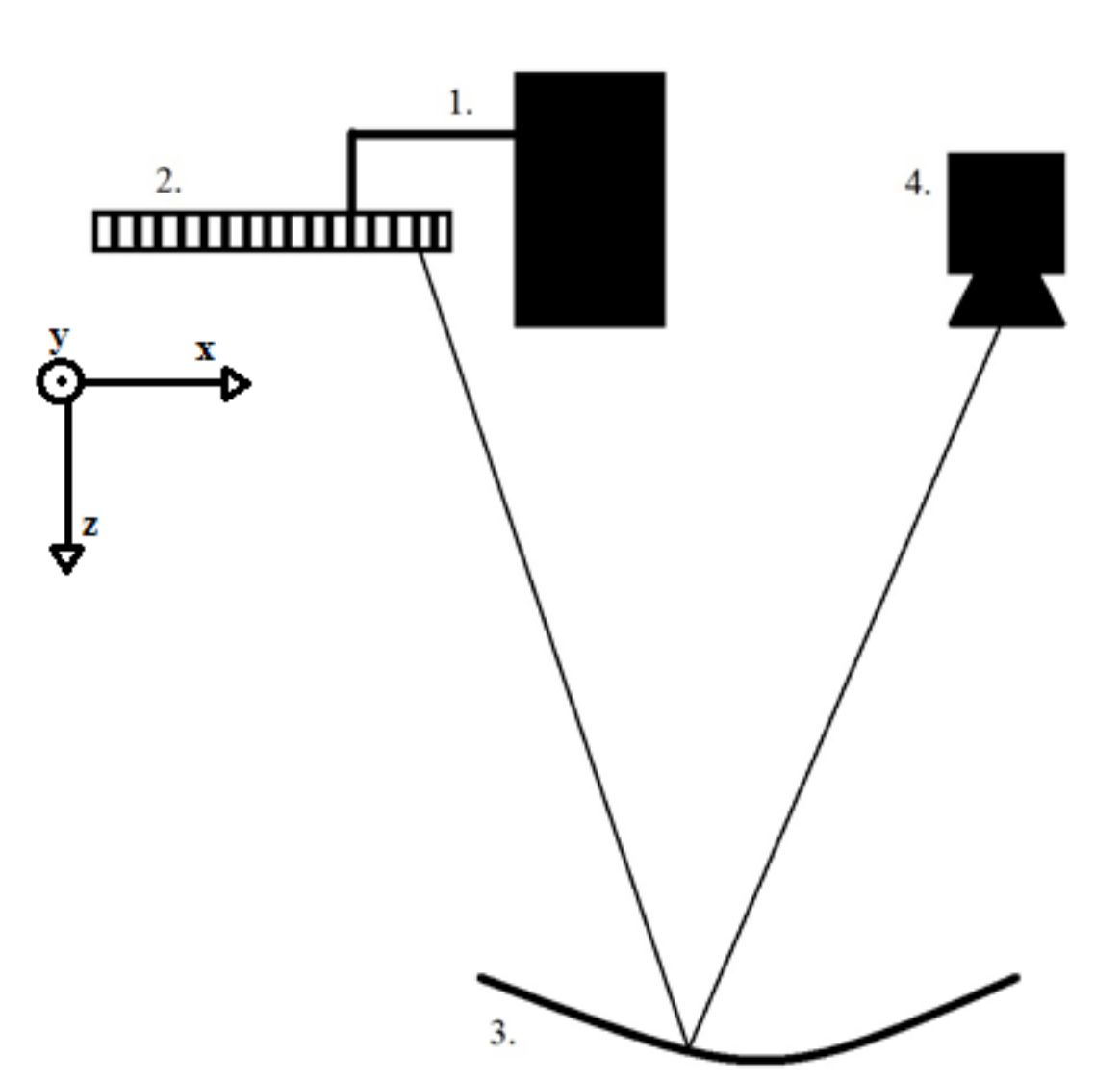

Figure 1. The schematic of the phase measuring
deflectometry (PMD) method A semera (4) observes the fringe pattern generated by computer (1) on a monitor (2) and reflected from the specular surface under the test (3). The observed fringe pattern is distorted. The figure of the surface can be derived distorted pattern. As it can be seen the observation angle does not have to be normal to the observation angle does
surface under the test.

Advantages of the PMD:
- It measures the surface slope directly

- It is relatively insensitive to vibrations,

No trace errors (does not need a compensator)

immune to coherent noise.

- It is fast, so many data sets can be averaged to reduce any random noise.

It has been demonstrated that the PMD method is capable of nanometer resolution 3,4 (on small, $100 \mathrm{~mm}$, flat samples and almost

at normal incidence with low resolution monitor-camera set)

it does not require the incidence (observation) angle to be normal for the measurements

does not require perfect positioning of the surface under test in the measuring system, so it can be adapted for in situ

Disadvantages

- It is slope measuring method - calibration errors in the case when the height profile needs to be resolved

Quality of fringe pattern defines the method resolution.

The reference phase map needs to be calculated for given surface under the test.

The highest resolution would be achieved at normal observation angle. The method resolution is lower for smaller observation angles

Experimental breadboard

A PMD breadboard have been developed. Photo of the breadboard is shown in Figure 4. In order to investigate the sensitivity of the method to low and mid-spatial-frequency surface errors three 4k cameras (Panasonic DMC GH4-YAGH Lumix DMC-GH4 4K Micro Four Thirds Digital Camera with DMW-YAGH 4K Video Interface) are mounted on stages. The positions of the cameras regarding the surface under the test can be varied. The cameras can be connected to the Blackmagic Design HyperDeck Studio Pro 2 recorder with solid state hard drives to speed up the image collection and file transfer. Depending on the surface spatial frequency under test three different lenses (Panasonic LUMIX G Leica DG Summilux 15mm f/1.7 ASPH. Lens, Panasonic Leica DG Summilux 25mm f/1.4 ASPH Micro 4/3 Lens, Olympus M.Zuiko Digital ED 75mm f/1.8 Lens) can be installed on the cameras. The sinusoidal fringe pattern is generated on the 4k monitor (Dell UItraSharp UP3214Q 31.5-Inch Screen LED-Lit Monitor).

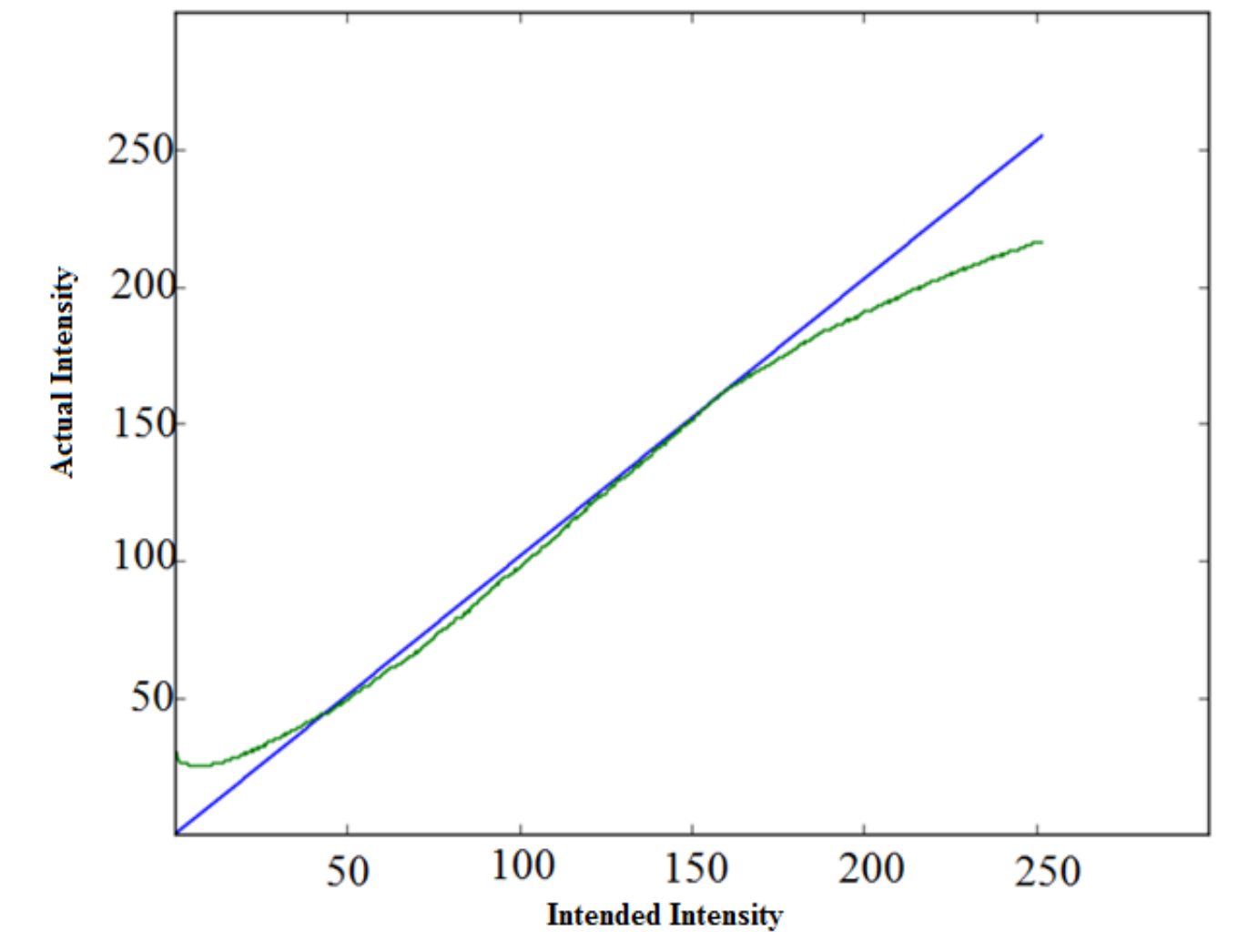

Figure 5. Actual monitor gray scale intensity (green) as captured by the camera. Blue line represents the intended
generated by computer PMD Breadboard calibration

To ensure high accuracy surface error measurements the monitor response and the camera lens distortions needs to be measured and calibrated out. Initial calibration was done using the ColorMunki calibrator at the center of the monitor. Then, the linear gray scale was generated at the center of computer monitor and one of the cameras was used to capture the gray scale image. The captured gray scale intensities are plotted on the Figure 5. This calibration curve was used to produce linear gray scale for each monitor pixel. The scale was also limited to the range from 60 to 220 to minimize the fringe distortions.

In order to define the global gray scale offset for the monitor the images of 160 uniform gray scale intensities were collected. Samples of the captured intensity maps are shown in figure 6 . The gray scale distortion pattern was calculated for each monitor pixel using these data. The average gray scale offset was calculated, the offset map is shown in figure 7. This map was used to correct global distortion of the gray scale on the monitor.

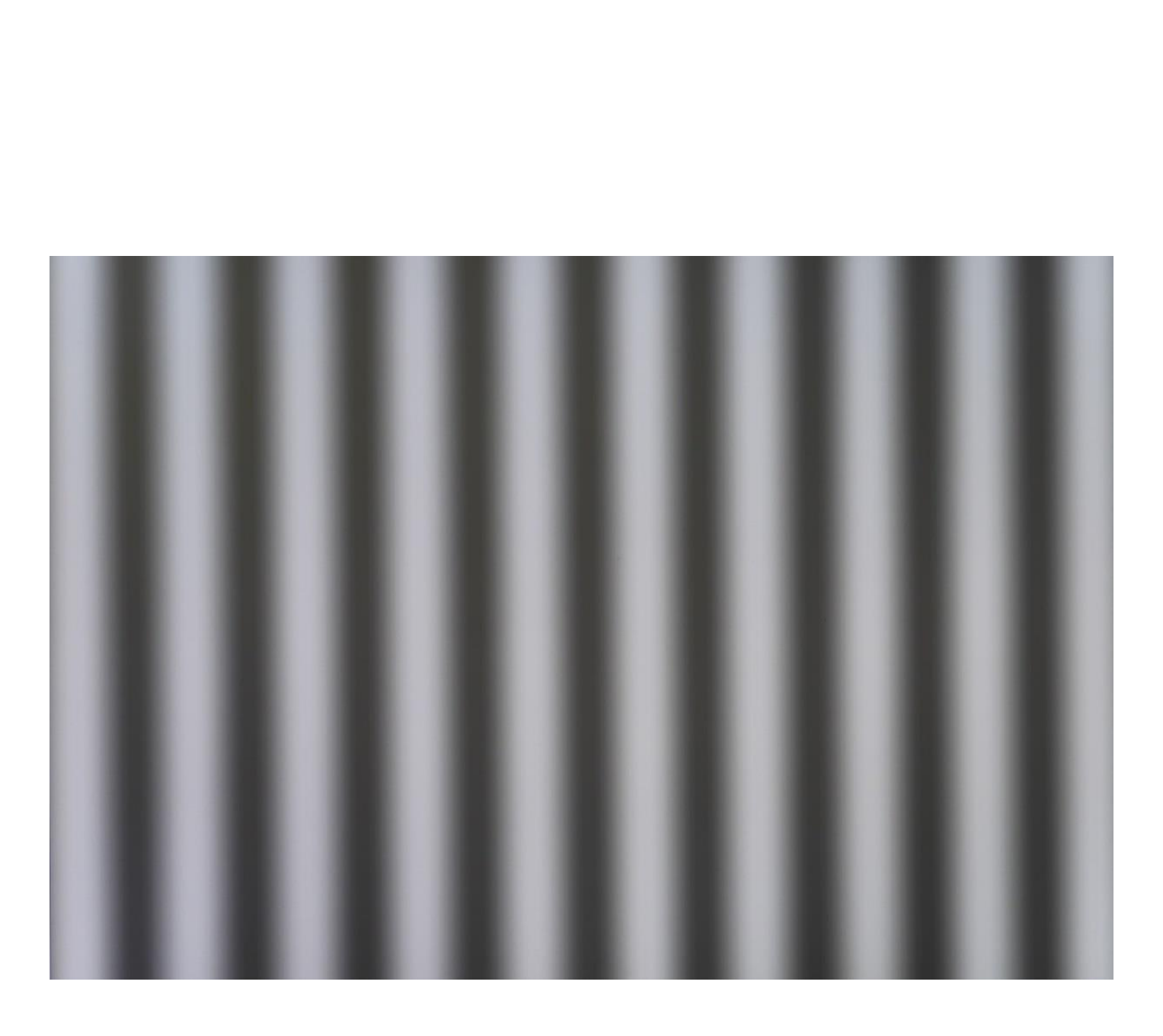

Figure 8. Sinusoidal fringe pattern generated
using gray scale and global offset calibration results.

\section{The sinusoidal fringe pattern was generated using the monitor}

data. The fringe pattern was captured by camera and

it is shown in Figure 8 . To demonstrate the improvement of the

atern aulity the captured and fitted fringe patterns with and

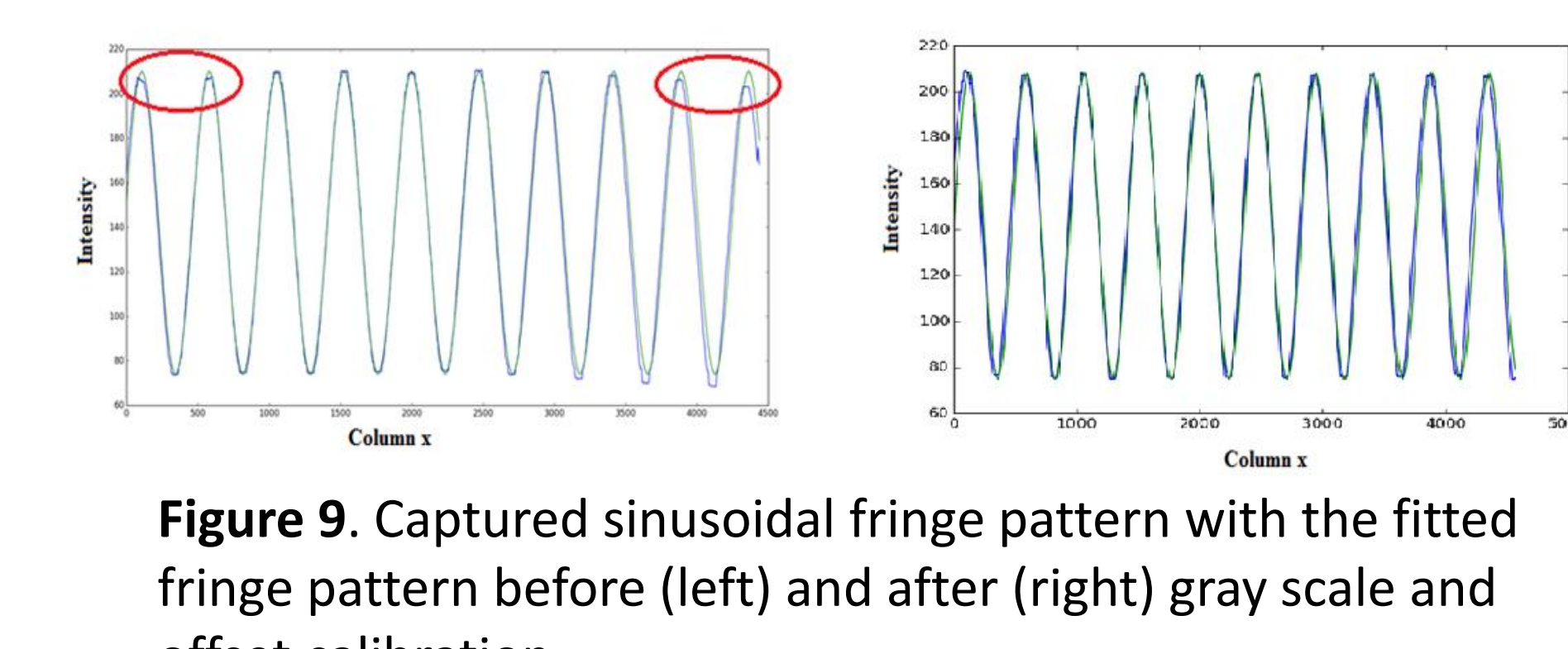

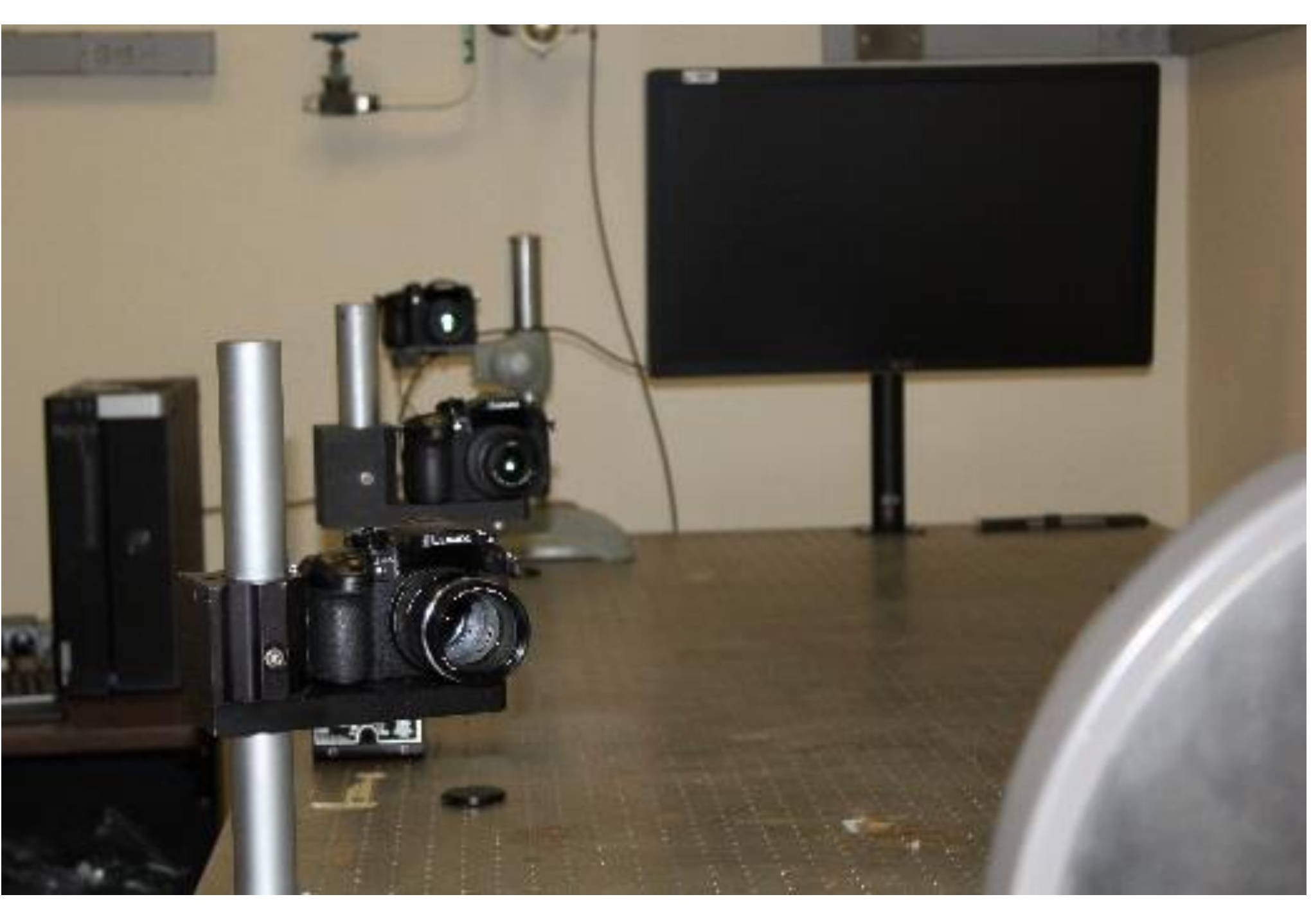

Figure 4. Experimental breadboard developed for the PMD studies. Three cameras positioned on an optical table collect the Interference pattern senerated by he mont or and reflected 